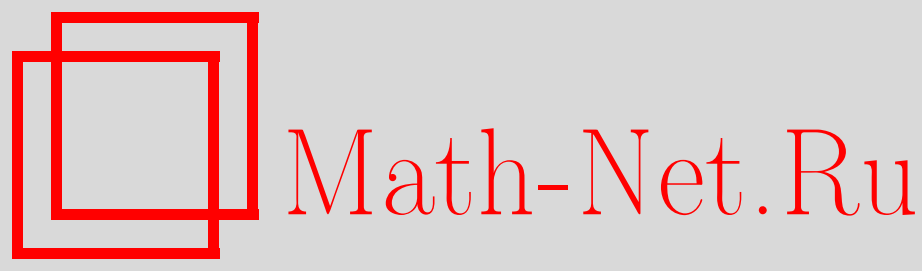

В. А. Москаленко, Л. А. Дохотару, Д. Ф. Дигор, И. Д. Чеботарь, Динамика фононных облаков коррелированных поляронов, ТМФ, 2014, том 179, номер 2, 242250

DOI: https://doi.org/10.4213/tmf8592

Использование Общероссийского математического портала Math-Net.Ru подразумевает, что вы прочитали и согласны с пользовательским соглашением http://www.mathnet.ru/rus/agreement

Параметры загрузки:

IP: 54.162 .127 .20

26 апреля 2023 г., 13:54:32

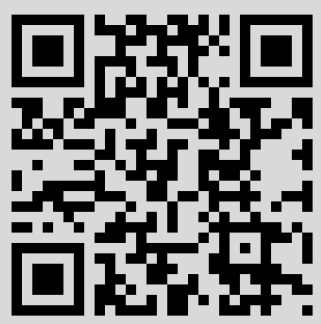




\title{
ФИЗИКА
}

Том 179, № 2

май, 2014

(C) 2014 г.

\author{
В. А. Москаленко ${ }^{* \dagger}$, Л. А. Дохотару \\ Д. Ф. Дигор*, И. Д. Чеботарь*

\section{ДИНАМИКА ФОНОННЫХ ОБЛАКОВ КОРРЕЛИРОВАННЫХ ПОЛЯРОНОВ}

\begin{abstract}
Исследована модель Андерсона-Холстейна с сильным отталкиванием примесных электронов в приближении сильной связи. Сильное электрон-фононное взаимодействие обусловливает появление поляронов с тяжелыми фононными облаками, окружающими примесные электроны. Описана коллективная мода фононных облаков и установлено соотношение между электронными пропагаторами и корреляционными функциями. Получена зависимость энергии коллективной моды от параметра гибридизации для случая $T=0$ и доказано ее подавление с ростом гибридизации.
\end{abstract}

Ключевые слова: сильно коррелированные электронные системы, уравнение Дайсона, функции Грина, полярон, фононные облака.

DOI: $10.4213 / \operatorname{tmf} 8592$

\section{1. ВВЕДЕНИЕ}

Эффект сильной корреляции в металлических системах может быть обусловлен как сильными электрон-электронными взаимодействиями, так и сильной электрон-фононной связью, в том числе их взаимным влиянием [1].

Существует множество систем с сильно коррелированными электронами, в которых также имеется сильная связь с решеткой, например $\mathrm{V}_{2} \mathrm{O}_{3}$ [2], [3], манганиты [4] и фуллериды [5]. Сильные электрон-электронные взаимодействия могут быть описаны с помощью моделей Хаббарда [6] и Андерсона [7]. Модель Холстейна [8] использовалась для изучения электрон-фононных взаимодействий. Модель Андерсона-Холстейна включает в себя оба вида взаимодействий.

* Институт прикладной физики АН Молдовы, Кишинев, Молдова.

E-mail: statphys@asm.md

† Объединенный институт ядерных исследований, Дубна, Московская обл., Россия. E-mail: moskalen@theor.jinr.ru

‡Технический университет Молдовы, Кишинев, Молдова.

E-mail: 1_dohotaru@mail.utm.md 
Достижения в области молекулярной электроники оживили интерес к задаче электрон-фононного взаимодействия, поскольку электрон-колебательная связь в молекуле играет важную роль для понимания свойств таких систем. С теоретической точки зрения это задача малого полярона, обсуждавшаяся Холстейном [8].

Гамильтониан примесной модели Андерсона-Холстейна имеет вид

$$
H=H_{\mathrm{el}}+H_{\mathrm{ph}}+H_{\mathrm{hyb}}+H_{\mathrm{el}-\mathrm{ph}},
$$

где

$$
\begin{aligned}
H_{\mathrm{el}} & =\sum_{\vec{k}, \sigma} \epsilon(\vec{k}) C_{\vec{k} \sigma}^{+} C_{\vec{k} \sigma}+\sum_{\sigma} \epsilon_{f}^{0} f_{\sigma}^{+} f_{\sigma}+U_{0} n_{\uparrow} n_{\downarrow}, \\
H_{\mathrm{ph}} & =\hbar \omega_{0}\left(a^{+} a+\frac{1}{2}\right), \quad q=\frac{1}{\sqrt{2}}\left(a+a^{+}\right), \quad p=\frac{i}{\sqrt{2}}\left(a^{+}-a\right), \\
H_{\mathrm{hyb}} & =\frac{1}{\sqrt{N}} \sum_{\vec{k}, \sigma}\left(V_{\vec{k}} f_{\sigma}^{+} C_{\vec{k} \sigma}+V_{\vec{k}}^{*} C_{\vec{k} \sigma}^{+} f_{\sigma}\right), \\
H_{\mathrm{el}-\mathrm{ph}} & =\bar{g} q n, \quad n=\sum_{\sigma} n_{\sigma}, \quad n_{\sigma}=f_{\sigma}^{+} f_{\sigma},
\end{aligned}
$$

где $q$ и $p$ - локальная координата и импульс оптических фононов, $\omega_{0}-$ их частота, $a\left(a^{+}\right), C_{\vec{k} \sigma}\left(C_{\vec{k} \sigma}^{+}\right)$и $f_{\sigma}\left(f_{\sigma}^{+}\right)$- операторы уничтожения (рождения) фононов, электронов проводимости и примесных электронов соответственно, $\epsilon(\vec{k})-$ кинетическая энергия электронов проводимости, $\epsilon_{f}^{0}$ - локальная энергия примесных электронов (обе величины энергии отсчитываются от химического потенциала $\mu_{0}$ системы: $\left.\epsilon(\vec{k})=\xi(\vec{k})-\mu_{0}, \epsilon_{f}^{0}=\bar{\epsilon}_{f}^{0}-\mu_{0}\right), U_{0}$ - одноузельное кулоновское отталкивание примесных электронов с противоположными спинами, $V_{\vec{k}}$ - матричный элемент гибридизации, $N$ - число узлов решетки, $\bar{g}$ - константа электрон-фононного взаимодействия. Обе величины $U_{0}$ и $\bar{g}$ слишком велики для того, чтобы рассматриваться как возмущение, поэтому их необходимо включить в нулевой гамильтониан $H_{0}=H_{\mathrm{el}}+H_{\mathrm{ph}}$.

Чтобы исключить линейный по $q$ член гамильтониана, выполним преобразование Ланга-Фирсова [9]:

$$
\begin{gathered}
\widetilde{H}=e^{S} H e^{-S}, \quad S=-i g n p, \quad g=\frac{\bar{g}}{\hbar \omega_{0}}, \\
\widetilde{a}=e^{S} a e^{-S}=a-\frac{n g}{\sqrt{2}}, \quad \widetilde{a}^{+}=e^{S} a^{+} e^{-S}=a^{+}-\frac{n g}{\sqrt{2}}, \\
\widetilde{q}=e^{S} q e^{-S}=q-n g, \quad n=n_{\uparrow}+n_{\downarrow}, \\
\widetilde{a}^{+} \widetilde{a}=a^{+} a-n g q+\frac{g^{2}}{2}\left(n_{\uparrow}+n_{\downarrow}+2 n_{\uparrow} n_{\downarrow}\right), \\
e^{S}\left(H_{\mathrm{ph}}+H_{\mathrm{el}-\mathrm{ph}}\right) e^{-S}=H_{\mathrm{ph}}-\frac{g^{2} \hbar \omega_{0}}{2}\left(n_{\uparrow}+n_{\downarrow}+2 n_{\uparrow} n_{\downarrow}\right), \\
\widetilde{H}_{\mathrm{el}-\mathrm{ph}}=\bar{g} n(q-n g) .
\end{gathered}
$$

Примесные операторы имеют вид

$$
\begin{gathered}
\tilde{f}_{\sigma}=e^{S} f_{\sigma} e^{-S}=e^{i g p} f_{\sigma}, \quad \tilde{f}_{\sigma}^{+}=f_{\sigma}^{+} e^{-i g p} \\
\widetilde{H}_{\mathrm{hyb}}=e^{S} H_{\mathrm{hyb}} e^{-S}=\frac{1}{\sqrt{N}} \sum_{\vec{k}, \sigma}\left(V_{\vec{k}} f_{\sigma}^{+} e^{-i g p} C_{\vec{k} \sigma}+V_{\vec{k}}^{*} C_{\vec{k} \sigma}^{+} f_{\sigma} e^{i g p}\right) .
\end{gathered}
$$

Операторы электронов проводимости остаются неизменными. 
В результате получаем

$$
\widetilde{H}=H^{0}+H_{\text {int }},
$$

где

$$
\begin{aligned}
H^{0} & =\sum_{\vec{k}, \sigma} \epsilon(\vec{k}) C_{\vec{k} \sigma}^{+} C_{\vec{k} \sigma}+\sum_{\sigma} \epsilon_{f} n_{\sigma}+U n_{\uparrow} n_{\downarrow}+\hbar \omega_{0}\left(a^{+} a+\frac{1}{2}\right), \\
H_{\mathrm{int}} & =\sum_{\sigma}\left(\widetilde{f}_{\sigma}^{+} b_{\sigma}+b_{\sigma}^{+} \widetilde{f}_{\sigma}\right)
\end{aligned}
$$

и

$$
\begin{gathered}
\epsilon_{f}=\epsilon_{f}^{0}-\mu, \quad \mu=\mu_{0}+\alpha \hbar \omega_{0}, \quad U=U_{0}-2 \alpha \hbar \omega_{0}, \quad \alpha=\frac{g^{2}}{2}, \\
b_{\sigma}=\frac{1}{\sqrt{N}} \sum_{\vec{k}} C_{\vec{k} \sigma} V_{\vec{k}}, \quad b_{\sigma}^{+}=\frac{1}{\sqrt{N}} \sum_{\vec{k}} V_{\vec{k}}^{*} C_{\vec{k} \sigma}^{+} .
\end{gathered}
$$

Оператор $b_{\sigma}\left(b_{\sigma}^{+}\right)$- локальный оператор электрона проводимости, $\tilde{f}_{\sigma}\left(\widetilde{f}_{\sigma}^{+}\right)-$оператор примесного электрона, окруженного фононами, т. е. полярона.

Гамильтониан $H^{0}$ есть главная часть $\widetilde{H}$, а $H_{\text {int }}$ - возмущение системы. Гамильтониан $H_{\text {int }}$ содержит как электронные, так и поляронные операторы. Благодаря присутствию сильного отталкивания $U$ нулевой гамильтониан $H^{0}$ описывает не только свободные частицы, а следовательно, обычная теорема Вика не может быть применена для всех электронов.

В развиваемом в дальнейшем подходе теории возмущений воспользуемся обобщенной теоремой Вика, предложенной в работах [10]-[14] для сильно коррелированных систем. Эта обобщенная теорема будет применена для подсистемы примесных электронов, а обычная теорема Вика - для электронов проводимости и оптических фононов.

В разделе 2 мы начинаем с ввода температурных мацубаровских функций Грина для электронов проводимости и примесных электронов в представлении взаимодействия. Раздел 3 посвящен анализу динамики фононных облаков. В разделе 4 обсуждаются полученные результаты и сделаны соответствующие выводы.

\section{2. ДИАГРАММНЫЙ ПОДХОД}

Мацубаровские перенормированные функции Грина электронов проводимости и примесных электронов в представлении взаимодействия определяются как

$$
\begin{aligned}
G\left(\sigma \tau \mid \sigma^{\prime} \tau^{\prime}\right) & =-\left\langle T b_{\sigma}(\tau) \bar{b}_{\sigma^{\prime}}\left(\tau^{\prime}\right) U(\beta)\right\rangle_{0}^{\mathrm{c}}, \\
g_{\mathrm{e}}\left(\sigma \tau \mid \sigma^{\prime} \tau^{\prime}\right) & =-\left\langle T f_{\sigma}(\tau) \bar{f}_{\sigma^{\prime}}\left(\tau^{\prime}\right) U(\beta)\right\rangle_{0}^{\mathrm{c}}, \\
g_{\mathrm{p}}\left(\sigma \tau \mid \sigma^{\prime} \tau^{\prime}\right) & =-\left\langle T \widetilde{f}_{\sigma}(\tau) \widetilde{\bar{f}}_{\sigma^{\prime}}\left(\tau^{\prime}\right) U(\beta)\right\rangle_{0}^{\mathrm{c}}
\end{aligned}
$$

где $\tau\left(\tau^{\prime}\right)$ обозначает мнимое время для $0<\tau<\beta, \beta$ - обратная температура системы, $T$ - хронологический оператор упорядочения. Статистическое усреднение $\langle\cdot\rangle_{0}^{\mathrm{c}}$ выполняется при помощи оператора плотности большого канонического ансамбля нулевого порядка $e^{-\beta H^{0} /\left(\operatorname{Tr} e^{-\beta H^{0}}\right)}$. Индекс “c" в (6) означает, что учитываются только связные диаграммы. 
Оператор эволюции $U(\beta)$ определяется как

$$
U(\beta)=T \exp \left(-\int_{0}^{\beta} H_{\mathrm{int}}(\tau) d \tau\right) .
$$

Определение (6) учитывает существование электронных $g_{\mathrm{e}}$ и поляронных $g_{\mathrm{p}}$ пропагаторов.

Кроме нормальных функций Грина (6), существуют аномальные функции Грина, определяемые как

$$
\begin{aligned}
F\left(\sigma \tau \mid-\sigma^{\prime} \tau^{\prime}\right) & =-\left\langle T b_{\sigma}(\tau) b_{-\sigma^{\prime}}\left(\tau^{\prime}\right) U(\beta)\right\rangle_{0}^{\mathrm{c}}, \\
\bar{F}\left(-\sigma \tau \mid \sigma^{\prime} \tau^{\prime}\right) & =-\left\langle T \bar{b}_{-\sigma}(\tau) \bar{b}_{\sigma^{\prime}}\left(\tau^{\prime}\right) U(\beta)\right\rangle_{0}^{\mathrm{c}}, \\
f_{\mathrm{e}}\left(\sigma \tau \mid-\sigma^{\prime} \tau^{\prime}\right) & =-\left\langle T f_{\sigma}(\tau) f_{-\sigma^{\prime}}\left(\tau^{\prime}\right) U(\beta)\right\rangle_{0}^{\mathrm{c}}, \\
f_{\mathrm{p}}\left(\sigma \tau \mid-\sigma^{\prime} \tau^{\prime}\right) & =-\left\langle T \widetilde{f}_{\sigma}(\tau) \widetilde{f}_{-\sigma^{\prime}}\left(\tau^{\prime}\right) U(\beta)\right\rangle_{0}^{\mathrm{c}}, \\
\bar{f}_{\mathrm{e}}\left(-\sigma \tau \mid \sigma^{\prime} \tau^{\prime}\right) & =-\left\langle T \bar{f}_{-\sigma}(\tau) \bar{f}_{\sigma^{\prime}}\left(\tau^{\prime}\right) U(\beta)\right\rangle_{0}^{\mathrm{c}}, \\
\bar{f}_{\mathrm{p}}\left(-\sigma \tau \mid \sigma^{\prime} \tau^{\prime}\right) & =-\left\langle T \widetilde{\bar{f}}_{-\sigma}(\tau) \widetilde{\bar{f}}_{\sigma^{\prime}}\left(\tau^{\prime}\right) U(\beta)\right\rangle_{0}^{\mathrm{c}},
\end{aligned}
$$

если система находится в сверхпроводящем состоянии.

Существуют также пропагаторы фононных облаков

$$
\begin{aligned}
\Phi\left(\tau_{1}-\tau_{2}\right) & =\left\langle T e^{i g\left(p\left(\tau_{1}\right)-p\left(\tau_{2}\right)\right)} U(\beta)\right\rangle_{0}^{\mathrm{c}}, \\
\varphi\left(\tau_{1}-\tau_{2}\right) & =\left\langle T e^{i g\left(p\left(\tau_{1}\right)+p\left(\tau_{2}\right)\right)} U(\beta)\right\rangle_{0}^{\mathrm{c}}, \\
\bar{\varphi}\left(\tau_{1}-\tau_{2}\right) & =\left\langle T e^{-i g\left(p\left(\tau_{1}\right)+p\left(\tau_{2}\right)\right)} U(\beta)\right\rangle_{0}^{\mathrm{c}}, \\
\Phi\left(\tau_{1} \tau_{2} \mid \tau_{3} \tau_{4}\right) & =\left\langle T e^{i g\left(p\left(\tau_{1}\right)+p\left(\tau_{2}\right)-p\left(\tau_{3}\right)-p\left(\tau_{4}\right)\right)} U(\beta)\right\rangle_{0}^{\mathrm{c}} .
\end{aligned}
$$

В дальнейшем будем полагать, что система находится в парамагнитном состоянии.

Используя обычную теорему Вика для фононных операторов, для нулевых пропагаторов $\Phi_{0}(\tau)$ и $\Phi_{0}\left(\tau_{1} \tau_{2} \mid \tau_{3} \tau_{4}\right)$ получаем [14]

$$
\begin{gathered}
\Phi_{0}\left(\tau_{1}-\tau_{2}\right)=e^{-g^{2}\left\langle T\left[p\left(\tau_{1}\right)-p\left(\tau_{2}\right)\right]^{2}\right\rangle_{0} / 2}=e^{-\sigma(\beta)+\sigma\left(\tau_{1}-\tau_{2}\right)}, \\
\Phi_{0}\left(\tau_{1} \tau_{2} \mid \tau_{3} \tau_{4}\right)=e^{-g^{2}\left\langle T\left[p\left(\tau_{1}\right)+p\left(\tau_{2}\right)-p\left(\tau_{3}\right)-p\left(\tau_{4}\right)\right]^{2}\right\rangle_{0} / 2}= \\
=e^{\sigma\left(\left|\tau_{1}-\tau_{3}\right|\right)+\sigma\left(\left|\tau_{1}-\tau_{4}\right|\right)+\sigma\left(\left|\tau_{2}-\tau_{3}\right|\right)+\sigma\left(\left|\tau_{2}-\tau_{4}\right|\right)-\sigma\left(\left|\tau_{1}-\tau_{2}\right|\right)-\sigma\left(\left|\tau_{3}-\tau_{4}\right|\right)-2 \sigma(\beta)},
\end{gathered}
$$

где

$$
\begin{aligned}
\sigma\left(\left|\tau_{1}-\tau_{2}\right|\right) & =g^{2}\left\langle T\left(p\left(\tau_{1}\right) p\left(\tau_{2}\right)\right)\right\rangle_{0}=\frac{\alpha \operatorname{ch}\left[\hbar \omega_{0}\left(\beta / 2-\left|\tau_{1}-\tau_{2}\right|\right)\right]}{\operatorname{sh}\left(\beta \hbar \omega_{0} / 2\right)} \\
\sigma(\beta) & =\sigma(0)=\alpha \operatorname{cth} \frac{\beta \hbar \omega_{0}}{2}
\end{aligned}
$$

Нам необходимо представление Фурье этих функций. Например,

$$
\Phi\left(\tau-\tau^{\prime}\right)=\frac{1}{\beta} \sum_{\Omega} e^{-i \Omega\left(\tau-\tau^{\prime}\right)} \Phi(i \Omega), \quad \Omega=\frac{2 n \pi}{\beta} .
$$


Представление Фурье пропагаторов фононных облаков (10), полученное с помощью приближения Лапласа в пределе сильной связи $\alpha \gg 1$, имеет вид [14], [15]

$$
\Phi_{0}(i \Omega) \approx \frac{2 \omega_{\mathrm{c}}}{\Omega^{2}+\omega_{\mathrm{c}}^{2}}, \quad \omega_{\mathrm{c}}=\alpha \omega_{0} .
$$

Этот член представляет собой гармонический пропагатор коллективной моды фононов, принадлежащих поляронному облаку с коллективной частотой $\omega_{\mathrm{c}}$. Это концепция свободных коллективных осцилляций фононных облаков, окружающих поляроны.

В нашем приближении другие два пропагатора $(9) \varphi\left(\tau, \tau^{\prime}\right)$ и $\bar{\varphi}\left(\tau, \tau^{\prime}\right)$ являются малыми величинами в нормальном состоянии системы, но они значительны в сверхпроводящем состоянии:

$$
\begin{aligned}
\varphi_{0}\left(\tau, \tau^{\prime}\right) & =\left\langle T e^{i g\left(p\left(\tau_{1}\right)+p\left(\tau_{2}\right)\right)}\right\rangle_{0}=e^{-g^{2}\left\langle T\left[p\left(\tau_{1}\right)+p\left(\tau_{2}\right)\right]^{2}\right\rangle_{0} / 2}= \\
& =e^{-\left(\sigma(\beta)+\sigma\left(\tau-\tau^{\prime}\right)\right)}, \quad \varphi_{0}\left(\tau, \tau^{\prime}\right) \sim e^{-\alpha} .
\end{aligned}
$$

Используемое нами для предела сильной связи приближение Лапласа $\alpha \gg 1$ позволяет доказать соотношение [14]

$$
\Phi_{0}\left(\tau_{1} \tau_{2} \mid \tau_{3} \tau_{4}\right) \approx \Phi_{0}\left(\tau_{1} \mid \tau_{3}\right) \Phi_{0}\left(\tau_{2} \mid \tau_{4}\right)+\Phi_{0}\left(\tau_{1} \mid \tau_{4}\right) \Phi_{0}\left(\tau_{2} \mid \tau_{3}\right)
$$

Это уравнение и его обобщение на многовременные аргументы рассматривается как теорема Вика для фононных облаков в режиме сильно связанных поляронов.

\section{3. ДИНАМИКА ФОНОННЫХ ОБЛАКОВ}

Исследуем процесс перенормировки пропагаторов фононных облаков, обусловленный гибридизацией электронных орбиталей. Во втором и четвертом порядках теории возмущений получим вклады, изображенные на рис. 1. Вклады от некоторых из диаграмм имеют следующий вид:

$$
\begin{aligned}
\Phi^{(2)}\left(\tau-\tau^{\prime}\right) & =\iint d \tau_{1} d \tau_{2} \Phi^{(0)}\left(\tau-\tau_{1}\right) \Pi_{2}\left(\tau_{1}-\tau_{2}\right) \Phi^{(0)}\left(\tau_{2}-\tau^{\prime}\right) \\
\varphi^{(2)}\left(\tau-\tau^{\prime}\right)= & \iint d \tau_{1} d \tau_{2} \Phi^{(0)}\left(\tau-\tau_{1}\right) \Pi_{2}^{s}\left(\tau_{1}-\tau_{2}\right) \Phi^{(0)}\left(\tau^{\prime}-\tau_{2}\right) \\
\Phi_{a}^{(4)}\left(\tau-\tau^{\prime}\right)= & \iiint \int d \tau_{1} d \tau_{2} d \tau_{3} d \tau_{4} \Phi^{(0)}\left(\tau-\tau_{1}\right) \times \\
& \times \Pi_{2}\left(\tau_{1}-\tau_{2}\right) \Phi^{(0)}\left(\tau_{2}-\tau_{3}\right) \Pi_{2}\left(\tau_{3}-\tau_{4}\right) \Phi^{(0)}\left(\tau_{4}-\tau^{\prime}\right),
\end{aligned}
$$

где

$$
\begin{aligned}
& \Pi_{2}\left(\tau_{1}-\tau_{2}\right)=-\left\langle T b_{1} \bar{b}_{2}\right\rangle_{0}\left\langle T f_{2} \bar{f}_{1}\right\rangle_{0} \\
& \Pi_{2}^{s}\left(\tau_{1}-\tau_{2}\right)=\left\langle T b_{1} b_{2}\right\rangle_{0}\left\langle T \bar{f}_{2} \bar{f}_{1}\right\rangle_{0}, \\
& \bar{\Pi}_{2}^{s}\left(\tau_{1}-\tau_{2}\right)=\left\langle T \bar{b}_{1} \bar{b}_{2}\right\rangle_{0}\left\langle T f_{2} f_{1}\right\rangle_{0} .
\end{aligned}
$$

В нормальном состоянии системы все аномальные функции Грина равны нулю, и мы полагаем, что малые пропагаторы $\varphi$ и $\bar{\varphi}$ также равны нулю. 


$$
\Phi^{(0)}\left(\tau-\tau^{\prime}\right)=
$$

Рис. 1. Перенормировка вкладов фононных облаков. Сплошная линия соответствует пропагатору электрона проводимости, а штриховая - пропагатору примесного электрона. Диаграммы изображают простейшие вклады второго и четвертого порядков теории возмущений. Петли представляют простейшие поляризационные операторы.

Диаграммы для нормального пропагатора $\Phi\left(\tau-\tau^{\prime}\right)$ могут быть суммированы в виде уравнения Дайсона

$$
\Phi\left(\tau-\tau^{\prime}\right)=\Phi^{(0)}\left(\tau-\tau^{\prime}\right)+\iint d \tau_{1} d \tau_{2} \Phi^{(0)}\left(\tau-\tau_{1}\right) \Pi\left(\tau_{1}-\tau_{2}\right) \Phi\left(\tau_{2}-\tau^{\prime}\right),
$$

где П $(\tau)$ - полный поляризационный оператор.

В представлении Фурье имеем

$$
\begin{aligned}
& \Phi(i \Omega)=\Phi^{(0)}(i \Omega)+\Phi^{(0)}(i \Omega) \Pi(i \Omega) \Phi(i \Omega) \\
& \Phi(i \Omega)=\frac{\Phi^{(0)}(i \Omega)}{1-\Phi^{(0)}(i \Omega) \Pi(i \Omega)}=\frac{1}{\left(\Phi^{(0)}(i \Omega)\right)^{-1}-\Pi(i \Omega)} .
\end{aligned}
$$


Используя выражение (14), получаем

$$
\Phi(i \Omega)=\frac{2 \omega_{\mathrm{c}}}{\Omega^{2}+\omega_{\mathrm{c}}^{2}-2 \omega_{\mathrm{c}} \Pi(i \Omega)} .
$$

Это уравнение определяет перенормировку коллективной фононной частоты $\omega_{\mathrm{c}}$. Новая частота определяется из уравнения

$$
E^{2}-\omega_{\mathrm{c}}^{2}+2 \omega_{\mathrm{c}} \Pi(E)=0
$$

Простейшие электронные функции Грина имеют вид $(\bar{\sigma}=-\sigma, \omega=(2 n+1) \pi / \beta)$

$$
G_{\sigma}^{0}(i \omega)=\frac{1}{N} \sum_{\vec{k}} \frac{\left|V_{\vec{k}}\right|^{2}}{i \omega-\epsilon(\vec{k})}, \quad g_{\sigma}^{0}(i \omega)=\frac{1-n_{-\sigma}}{\lambda_{\sigma}(i \omega)}+\frac{n_{-\sigma}}{\bar{\lambda}_{\bar{\sigma}}(i \omega)}
$$

где

$$
\begin{gathered}
\lambda_{\sigma}(i \omega)=i \omega+E_{0}-E_{\sigma}, \quad E_{0}=0, \quad E_{\sigma}=\epsilon_{f}, \quad E_{2}=U+2 \epsilon_{f}, \\
\bar{\lambda}_{\bar{\sigma}}(i \omega)=i \omega+E_{-\sigma}-E_{2}, \quad Z_{0}=e^{-\beta E_{0}}+2 e^{-\beta E_{\bar{\sigma}}}+e^{-\beta E_{2}}, \\
n_{\bar{\sigma}}=\frac{e^{-\beta E_{\bar{\sigma}}}+e^{-\beta E_{2}}}{Z_{0}}, \quad 1-n_{\bar{\sigma}}=\frac{e^{-\beta E_{0}}+e^{-\beta E_{\sigma}}}{Z_{0}} .
\end{gathered}
$$

Эти функции определяют простейший поляризационный оператор $\Pi_{2}(i \Omega)$ в нормальном состоянии:

$$
\Pi_{2}(i \Omega)=-\frac{1}{\beta} \sum_{\omega} G_{\sigma}^{0}(i \omega) g_{\sigma}^{0}(i \omega-i \Omega)
$$

Суммирование по частотам приводит к следующему результату:

$$
\begin{aligned}
\Pi_{2}(i \Omega)=\frac{1}{N} \sum_{\vec{k}, \sigma}\left|V_{\vec{k}}\right|^{2}\left\{\frac{1-n_{\bar{\sigma}}}{i \Omega-\epsilon(\vec{k})+\epsilon_{f}}\left[\frac{1}{e^{\beta \epsilon(\vec{k})}+1}-\frac{1}{e^{\beta \epsilon_{f}}+1}\right]+\right. \\
\left.+\frac{n_{\bar{\sigma}}}{i \Omega-\epsilon(\vec{k})+U+\epsilon_{f}}\left[\frac{1}{e^{\beta \epsilon(\vec{k})}+1}-\frac{1}{e^{\beta\left(U+\epsilon_{f}\right)}+1}\right]\right\} .
\end{aligned}
$$

Для выполнения суммирования по вектору $\vec{k}$ перейдем к интегрированию по энергиям:

$$
\frac{1}{N} \sum_{\vec{k}}(\cdot) \longrightarrow \int(\cdot) \rho(E) d E
$$

где $\rho(E)$ - плотность состояний.

С помощью уравнения (22) для случая $T=0$ при

$$
\rho(E)=\frac{1}{2 W} \begin{cases}1, & E<|W| \\ 0, & E>|W|\end{cases}
$$




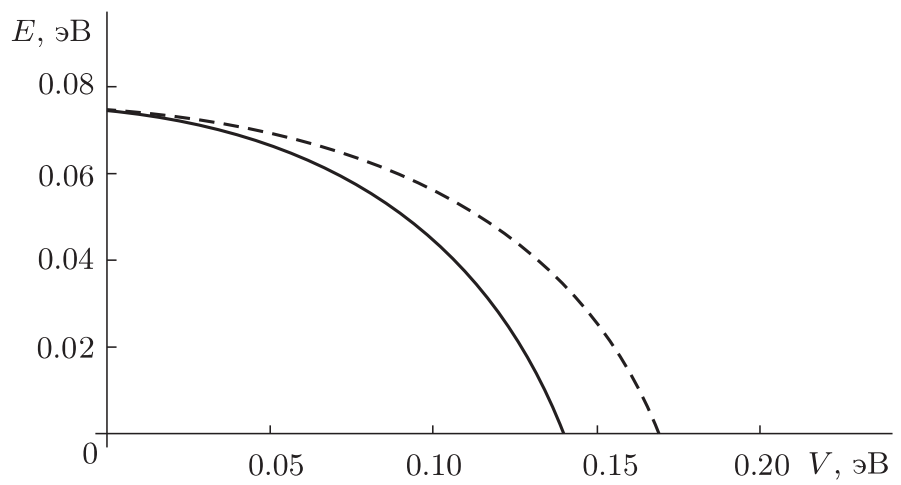

Рис. 2. Энергия коллективной моды $E$ в зависимости от параметра гибридизации $V$ для различных значений ширины зоны: $W=0.5$ эВ (сплошная линия) и $W=1$ эВ (штриховая линия). Другие параметры приняты следующими: $\omega_{\mathrm{c}}=0.075$ эВ $U=5.85$ э $\mathrm{B}, \epsilon_{\mathrm{F}}=-0.095$ эВ.

где $W$ - ширина зоны, мы исследовали зависимость энергии коллективной моды $E$ относительно параметра гибридизации $V$. Результаты численных расчетов представлены на рис. 2.

Согласно этим результатам перенормированная энергия коллективной моды $E$ фононных облаков уменьшается с увеличением величины $V$ и становится равной нулю при критическом значении

$$
V_{\mathrm{c}}=\sqrt{\frac{\hbar \omega_{\mathrm{c}} W}{\ln \left|\left(W-\epsilon_{f}\right)\left(W+U+\epsilon_{f}\right) /\left(\epsilon_{f}\left(U+\epsilon_{f}\right)\right)\right|}} .
$$

Очевидно, что для больших значений ширины энергетической зоны $W$ критическое

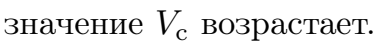

Таким образом, мы делаем вывод, что коллективная мода подавляется гибридизацией.

\section{4. ВЫВОДЫ}

Развит диаграммный подход для модели Андерсона-Холстейна в предположении сильного кулоновского межатомного взаимодействия примесных электронов и при наличии сильной связи электрон-фононного взаимодействия этих электронов с оптическими фононами. Оба взаимодействия считаются сильными и учитываются в главной части гамильтониана.

Исследована динамика фононных облаков поляронов, подробно описана перенормировка их коллективной частоты.

Доказано, что при $T=0$ коллективная мода подавляется, если гибридизация становится сильной.

Благодарности. Авторы благодарны С. Кожокару за очень полезное обсуждение. 


\section{Список литературы}

[1] W. Koller, D. Meyer, Y. Ōno, A. C. Hewson, Europhys. Lett., 66:4 (2004), 559-564, arXiv: cond-mat/0312367.

[2] P. Majumdar, H. R. Krishnamurthy, Phys. Rev. Lett., 73:11 (1994), 1525-5128.

[3] M. Imada, A. Fujimori, Y. Tokura, Rev. Modern Phys., 70:4 (1998), 1039-1263.

[4] A. J. Millis, P. B. Littlewood, B. J. Shraiman, Phys. Rev. Lett., 74:25 (1995), 5144-5147.

[5] O. Gunnarsson, Rev. Modern Phys., 69:2 (1997), 575-606.

[6] J. H. Hubbard, Proc. R. Soc. London Ser. A, 276:1365 (1963), 238-257; 281:1386 (1964), 401-419; 285:1403 (1965), 542-560.

[7] P. W. Anderson, Phys. Rev., 124:1 (1961), 41-53.

[8] T. Holstein, Ann. Phys., 8:3 (1959), 343-389.

[9] I. G. Lang, Yu. A. Firsov, ЖKЭТФ, 43 (1962), 1843-1860.

[10] М. И. Владимир, В. А. Москаленко, ТМФ, 82:3 (1990), 428-437.

[11] С. И. Вакару, М. И. Владимир, В. А. Москаленко, ТМФ, 85:2 (1990), 248-257.

[12] Н. Н. Боголюбов, В. А. Москаленко, ТМФ, 86:1 (1991), 16-30.

[13] Н. Н. Боголюбов, В. А. Москаленко, ТМФ, 92:2 (1992), 182-190.

[14] V. A. Moskalenko, P. Entel, D. F. Digor, Phys. Rev. B, 59:1 (1999), 619-635.

[15] В. А. Москаленко, ТМФ, 111:3 (1997), 439-451; 113:3 (1997), 432-437.

Поступила в редакцию 4.09.2013, после доработки 20.11.2013 